\title{
Editorial
}

\section{Analytical and Numerical Approaches for Complicated Nonlinear Equations}

\author{
Hossein Jafari, ${ }^{1}$ Abdon Atangana, ${ }^{2}$ Adem Kılıçman, ${ }^{3}$ and Muhammad Usman ${ }^{4}$ \\ ${ }^{1}$ Department of Mathematics, University of Mazandaran, Babolsar 47416-95447, Iran \\ ${ }^{2}$ Department of Applied Mathematics, Institute for Groundwater Studies, University of the Free State, Bloemfontein 9301, South Africa \\ ${ }^{3}$ Department of Mathematics and Institute for Mathematical Research, University Putra Malaysia, 43400 Serdang, Selangor, Malaysia \\ ${ }^{4}$ Department of Mathematics, University of Dayton, College Park 300, Dayton, OH 45469-2316, USA
}

Correspondence should be addressed to Hossein Jafari; jafari@umz.ac.ir

Received 7 September 2014; Accepted 7 September 2014; Published 21 December 2014

Copyright (C) 2014 Hossein Jafari et al. This is an open access article distributed under the Creative Commons Attribution License, which permits unrestricted use, distribution, and reproduction in any medium, provided the original work is properly cited.

\begin{abstract}
Mathematical tools have been intensively used for the description of many natural phenomena. More often, these natural phenomena are first observed and converted into a mathematical formula usually called equations. One of the most obtained classes of equations is the nonlinear type. In order to predict the future behavior of the considered natural phenomena, we solve these equations either numerically or analytically. No wonder why many researchers in this field have devoted their attention to proposing numerical and analytical methods for these classes of equations. The aim of the special issue was to collect all the latest results and improvements done in this field. We have received 67 very good research papers; in order to keep the standard very high we have selected only 36 .

This issue includes papers on dissimilar features, for example, new classes of boundary value conditions for fractional differential equations, singular differential equations, controllability of partial differential equations, bifurcation of positive solutions, impulsive problems, inverse problems, and numerical methods using, for example, recursively compressed inverse preconditioning, finite element method, or hybrid topological derivative-gradient-based methods and iterations methods.

Obviously, it is not conceivable to sufficiently characterize in this special issue all guidelines of up-to-date investigation on nonlinear problems; nonetheless we trust that it reproduces both theoretical research and important recent advances including current challenging problems, new ideas, and open problems.
\end{abstract}

\section{Acknowledgment}

We recognize all the contributors to this special issue for their participation.

Hossein Jafari Abdon Atangana Adem Kılıçman Muhammad Usman 


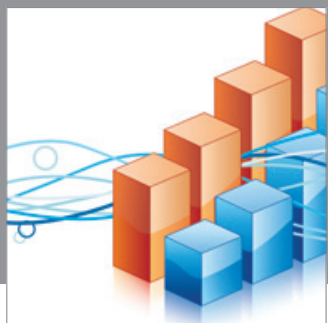

Advances in

Operations Research

mansans

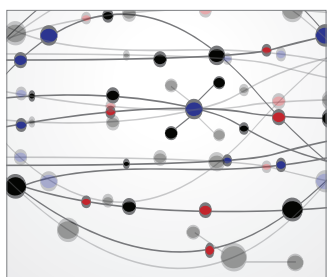

The Scientific World Journal
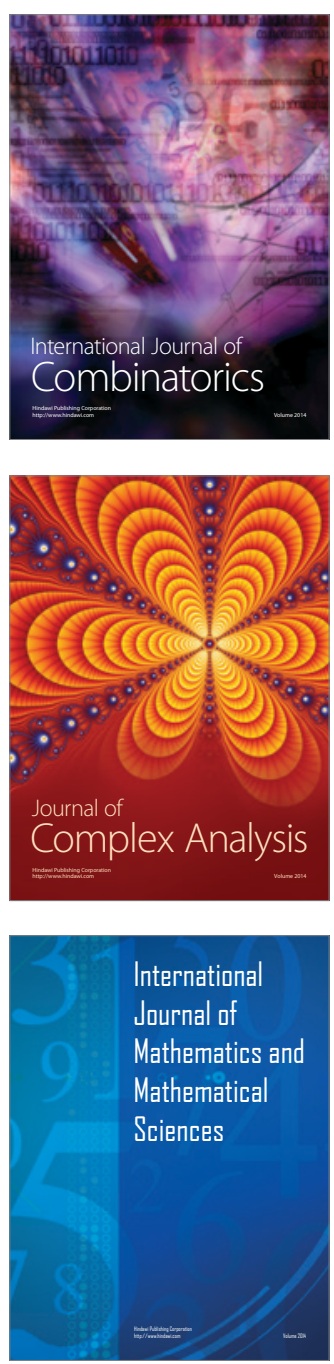
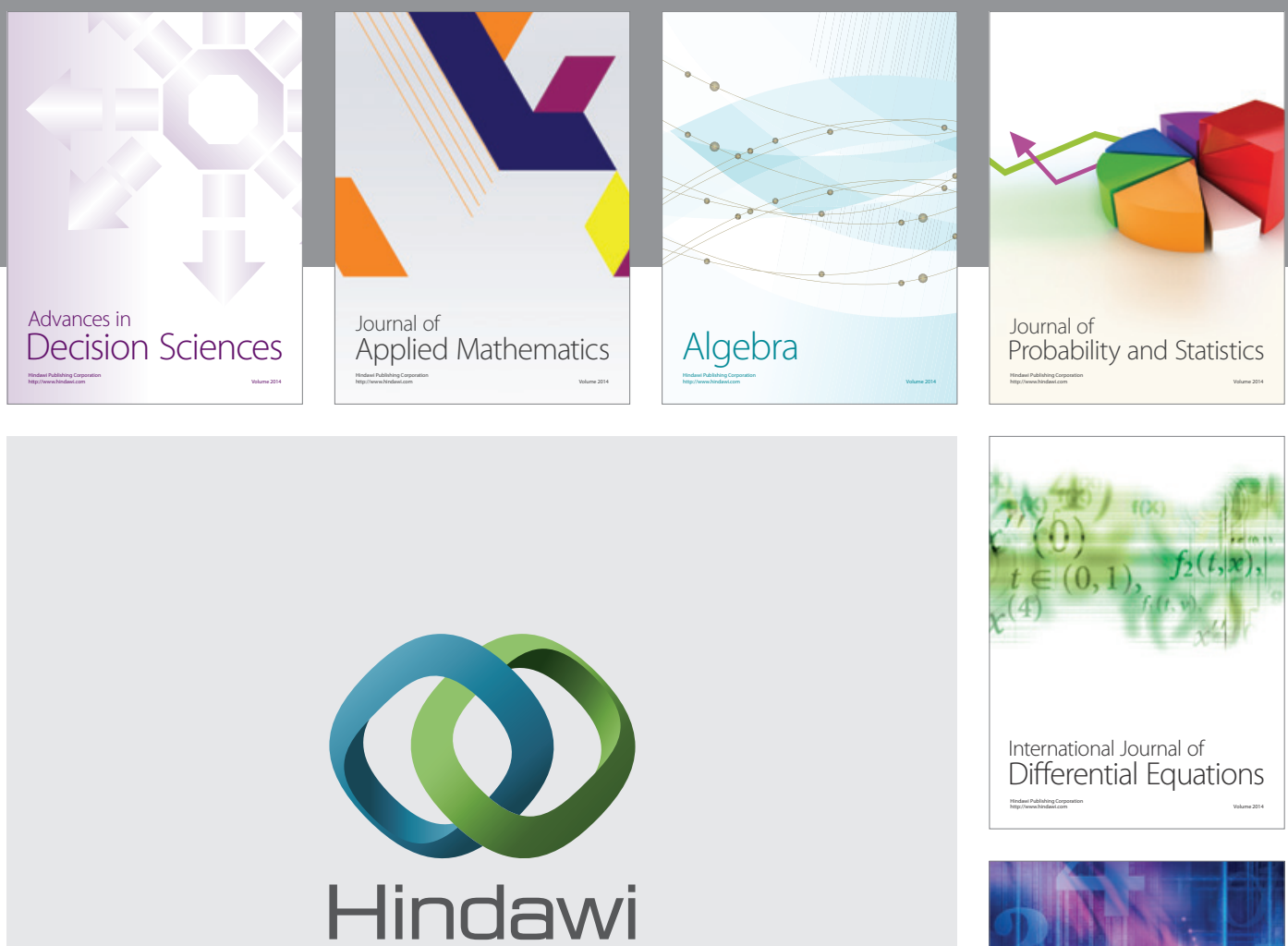

Submit your manuscripts at http://www.hindawi.com
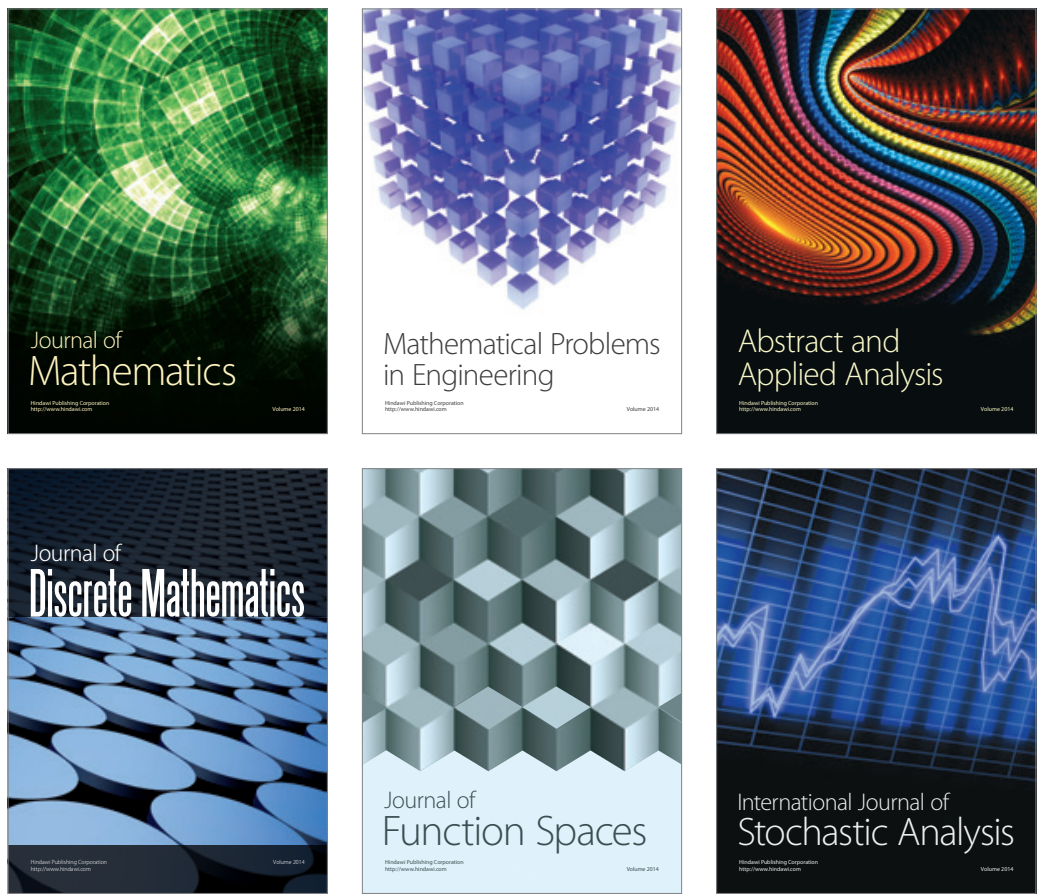

Journal of

Function Spaces

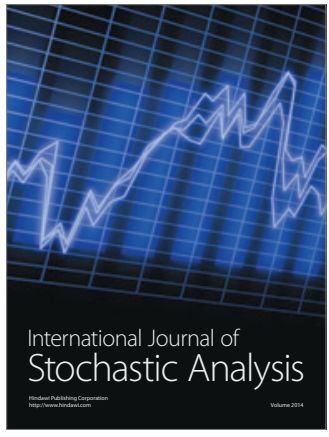

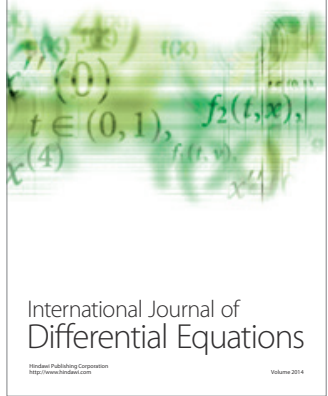
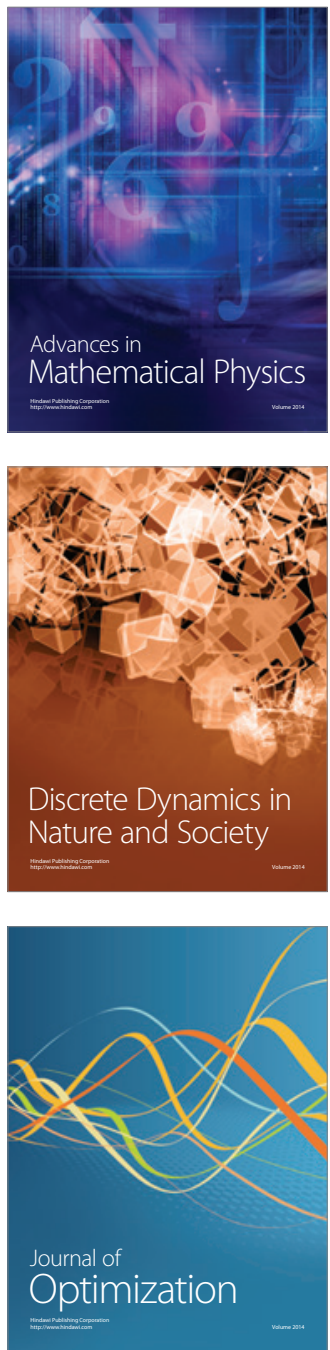